\title{
On numerical methods of finding an approximate solution of multi-parameters eigenvalue problems
}

\author{
Yaroshko S. ${ }^{1}$, Yaroshko O. ${ }^{2}$ \\ ${ }^{1}$ Lviv Polytechnic National University \\ 12 S. Bandera str., 79013, Lviv, Ukraine \\ ${ }^{2}$ Ivan Franko National University of Lviv \\ 1 Universytetska str., 79000, Lviv, Ukraine
}

(Received 4 October 2017)

\begin{abstract}
This survey is intended to give an overview of the existing numerical methods and techniques for solving a multi-parameter eigenvalue problem. It also collects some well-known examples of multi-parameter spectral problem and its practical applications in different scientific areas.
\end{abstract}

Keywords: eigenvalue, eigenvector, eigenvalue problem, multi-parameter spectral problem, numerical methods.

2000 MSC: 34B09, 34L15, 34L16, 58C40, 65F15

UDC: 519.6

DOI: $10.23939 / \mathrm{mmc} 2017.02 .197$

\section{Application of multi-parameter spectral problems}

Consider an operator equation

$$
T(\lambda) x=f
$$

where $T(\lambda): R^{n} \rightarrow L(H)$ is an operator function, $L(H)$ is a set of linear operators, that act in a Hilbert space $H$. The function $T(\lambda)$ depends on two or more spectral parameters $\lambda=\left(\lambda_{1}, \lambda_{2}, \ldots, \lambda_{n}\right), n \geqslant 2$, in a linear or a nonlinear way.

The analysis of solvability of the equation (1) involves a problem of finding such values of the parameters $\lambda_{i}, i=1,2, \ldots, n$, that there exists a non-trivial solution of the following homogeneous equation:

$$
T(\lambda) x=0
$$

Such problems are known as two- or multi-parameter spectral problems, and they arise in different areas of scientific and engineering applications, in some boundary-value problems, and in the problems of applied mathematics and functional analysis.

In general, the applied problems which lead to the multi-parameter spectral problems, can be divided into three main categories [1]. The first one, the widest from the point of view of practical application, consists of the oscillation analysis problems. Usually, these are the symmetric generalized eigenvalue problems that derive from this category. The second category is the class of stability analysis problems. Such problems generate the spectral problem for non-symmetric matrices. The third category includes different problems of physics, related to the quantum mechanical oscillations.

A wide range of matrix spectral problems occurs in numerical modeling of chemical reactions. An interesting class of such reactions is one, where the periodic reactions arise spontaneously. A known example of such type of reaction is the Belousov-Zhabotinski reaction, described by so-called Brusselator model [1].

As an example of two-parameters spectral problem, let us consider the following case [2]:

$$
\begin{aligned}
& A_{1} x_{1}=\lambda B_{1} x_{1}+\mu C_{1} x_{1}, \\
& A_{2} x_{2}=\lambda B_{2} x_{2}+\mu C_{2} x_{2},
\end{aligned}
$$


where the given real- or complex-valued matrices $A_{1}, B_{1}, C_{1}$ are of dimension $n_{1} \times n_{1}$, and the similar matrices $A_{2}, B_{2}, C_{2}$ are of the size $n_{2} \times n_{2}$.

The problem consists in finding the eigen pairs $\left((\lambda, \mu), x_{1} \otimes x_{2}\right)$ such that the eigenvectors $x_{1}$ and $x_{2}$ have a unit norm.

Obviously, this two-parameters eigenvalue problem can be easily extended to a multi-parameter case. The multi-parameter problems of this type arise in a wide range of applications, for example in the problems of mathematical physics, when applying a method of splitting the variables to solve some boundary problems.

One of the first applications of a multi-parameter eigenvalues problem can be found in the SturmLiouville theory [3]. The multi-parameter analogue of the classic Sturm-Liouville problem can be formulated as follows. There are given $k$ differential equations:

$$
y_{r}^{\prime \prime}\left(x_{r}\right)+\left(\sum_{s=1}^{k} \lambda_{s} p_{r s}\left(x_{r}\right)+q_{r}\left(x_{r}\right)\right) y_{r}\left(x_{r}\right)=0 .
$$

It is required to find $k$ functions $y_{r}\left(x_{r}\right), a_{r} \leqslant x_{r} \leqslant b_{r}$ such that they satisfy the differential equations (3).

For the boundary problem let us define the parameters $\lambda_{1}, \ldots, \lambda_{k}$ such that each equation (3) has a non-trivial solution satisfying the boundary conditions:

$$
y_{r}\left(a_{r}\right)=y_{r}\left(b_{r}\right)=0, \quad r=1, \ldots, k .
$$

In order to ensure that all the eigenvalues are real, an extra requirement is added: the parameters $p_{r s}, q_{r}$ must be real, and the following conditions are fulfilled:

$$
\operatorname{det}\left\{p_{r s}\left(x_{r}\right)\right\}_{r, s=1}^{k}>0, \quad a_{r} \leqslant x_{r} \leqslant b_{r}, \quad r=1, \ldots, k .
$$

An interesting example of boundary spectral problem for a differential equation is one for the following linear differential equation [4]:

$$
\left\{\begin{array}{l}
\sum_{j=0}^{k} a_{j}(\mu, x) y^{(j)}(x)=\lambda \sum_{j=0}^{k-1} b_{j}(\mu, x) y^{(j)}(x), \quad x \in[a, b] \\
\left(L^{j} y\right)(a) \cdot \mid \cdot\left(M^{j} y\right)(b)=0, \quad j=1, \ldots, k
\end{array}\right.
$$

where $a_{j}(\mu, x), b_{j}(\mu, x)$ are the real-valued functions of $\mu \in\left(\mu_{a}, \mu_{b}\right) \subset R$, and $L^{j}, M^{j}$ are the linear differential operators. The most known example of the problem (5) is the differential equation of Mathieu.

The multi-parameter eigenvalue problem is widely used in solving the engineering problems, for example, the induction machine initial conditions problem and the power flow equation [5]. Based on the fact that the eigenvalue problem incorporates a lot of non-linearities into the linear equations, the engineering models can be reformulated as a spectral problem. Indeed, the classic eigenvalue problem $A x=\lambda x$ includes only one non-linearity - the multiplication of the eigenvector $x$ to the eigenvalue $\lambda$. The multi-parameter spectral problem with $\mathrm{n}$ parameters includes $n$ multiplicative non-linearities. Thus, the formulation of the engineering problems in terms of an eigenvalue problem is interesting from the theoretical point of view. Moreover, it has an important impact for the practical usage, because this allows one to apply the numerical method for spectral problems to find the solution of the original engineering problem.

Certainly, the presented list of applications of the spectral problems is not complete. Moreover, the number of practical applications of the eigenvalue problems constantly grows. 


\section{Numerical methods for multi-parameter spectral problem}

A lot of numerical methods for multi-parameter spectral problem are known. Some of them provide just one approximate eigenvalue, while other methods calculate all the eigenvalues of the given matrix. The first type of methods are the direct methods, and they can be found in the works of Bloom, Bohte, Browne, Sleeman. The specificity of these methods is that they are effective only for the matrices of small dimensions. The second type of methods are the continuous methods, which are less expensive than the direct methods, but still not effective enough even for the matrices of small sizes.

Frequently, on practice it is needed to find only a couple of relevant eigen pairs. Those can be, for example, the pairs corresponding to the biggest eigenvalue. For such kind of multi-parameter problems there were provided some specific methods of subspace, which are based on applying the subspace approach in combination with a dense method. For example, as one type of the dense methods, the harmonic extraction methods [2] can be. These methods are designed for multi-parameter spectral problem so, that they find the eigen pair in the specific subspace, and the found approximation is as close to the exact eigen pair, as possible.

Another wide range of numerical methods unify the methods of spectral approximation [1]. A lot of approximation algorithms of the matrix spectrum combine different mathematical tools: the projection method, the acceleration Chebyshev method, the offset and invert strategies, etc. As soon as one eigen pair is found, arise the question of calculation of the next pair. This goal can be achieved by using the standard technique, known as the deflation technique.

One of the oldest methods of solving the eigenvalue problems is the power method. The idea of power method is to generate the sequence of vectors $A^{k} v_{0}$, where $v_{0}$ is a non-trivial zero approximation vector. The advantage of this method is its simplicity. However, the convergence rate can be too slow in some cases.

In order to improve the convergence rate of standard methods, different techniques can be applied. For example, the polynomial filtering methods are used to accelerate the convergence of classic numerical methods for eigenvalues and eigenvectors. These methods use the polynomial iterations $z_{q}=p_{q}(A) z_{0}$, where $p_{q}$ is a polynomial of power $q$, constructed based on the information about the distribution of eigenvalues of the matrix $A$.

The main goal of the polynomial filtering is to enforce the projection scheme by using the starting vectors or the starting subspaces so that to decrease the number of their components in the undesired parts of spectrum, versus the components in the desired parts of spectrum.

Suppose that there is provided the Hermite matrix $A$ with the eigenvalues $\lambda_{1}>\lambda_{2} \geqslant \ldots \geqslant \lambda_{n}$ and the corresponding orthogonal eigenvectors $u_{1}, \ldots, u_{n}$.

If we would be interested in the major eigenvalue $\lambda_{1}$, then we could apply the power method, under the assumption that $\left|\lambda_{1}\right|>\left|\lambda_{i}\right|$ for $i>1$. This means that to calculate the eigenvalues there will be used the polynomial $p_{q}(t)=t^{q}$. But it is interesting to use some other polynomial - the one which will allow to improve the convergence of the method. In other words, we're interested in the iteration like $x_{q}=p_{q}(A) x_{0}$.

Unfolding the eigenvector $x_{0}$ by the eigen basis results in

$$
x_{0}=\sum_{i=1}^{n} \gamma_{i} u_{i} .
$$

Consequently,

$$
\begin{aligned}
x_{q} & =p_{q}(A) x_{0}=\sum_{i=1}^{n} p_{q}\left(\lambda_{i}\right) \gamma_{i} u_{i} \\
& =p_{q}\left(\lambda_{1}\right) \gamma_{1} u_{1}+\sum_{i=2}^{n} p_{q}\left(\lambda_{i}\right) \gamma_{i} u_{i}
\end{aligned}
$$

Mathematical Modeling and Computing, Vol.4, No. 2, pp. 197-205 (2017) 
In case it is needed to find the first eigen pair, we can require that in (6) the component $p_{q}\left(\lambda_{1}\right) \gamma_{1}$ is bigger than the other components $p_{q}\left(\lambda_{i}\right) \gamma_{i}, i>1$. If this property is true, it will be needed to scale the polynomial $p_{q}$, for example, $p_{q}\left(\lambda_{1}\right)=1$. On practice the value $\lambda_{1}$ is not known, but the scaling is done based on the approximation $\tilde{\lambda}_{1}$.

The parameters $\gamma_{i}$ usually are not known either. Thus, we are looking for the polynomial $p_{q}$, whose absolute maximum value is the smallest one between the values of the polynomial in the points $\lambda_{i}$, $i>1$. As far as the values $\lambda_{i}$ are unknown, a more realistic task will be to find the polynomial $p_{q}$, whose value in the point $\lambda_{1}$ is equal to 1 , and the absolute maximum value on the interval $[\alpha, \beta]$ of all other eigenvalues is as small as possible. The mathematical formulation of this problem can be described as follows:

$$
\min _{\substack{p_{q} \in P_{q} \\ p_{q}\left(\lambda_{1}\right)=1}} \max _{t \in[\alpha, \beta]}\left|p_{q}(t)\right| .
$$

The optimal polynomial is the scaled Chebyshev polynomial of first kind of the power $q$ :

$$
C_{q}(t) \equiv C_{q}\left(1+2 \frac{t-\beta}{\beta-\alpha}\right) / C_{q}\left(1+2 \frac{\lambda_{1}-\beta}{\beta-\alpha}\right) .
$$

Using this polynomial it is easy to construct the final formula of calculating the next approximation of the eigenvector

$$
x_{q}=\widehat{C}_{q}(A) x_{0}
$$

The major part of algorithms of solving the spectral problems involves the projection technique. The projection process can be the base of the method as well as just a part of a complex algorithm, needed to increase the efficiency of this algorithm. A simple example of the need of applying the projection technique is the case, when the power method is applied to solve a spectral problem with complex-valued dominant eigenvalue and real-valued matrix [1].

There is an entire class of methods for the spectral problems with the matrices of big dimensions. The simplest one in this class certainly is the method of subspace iteration, which can be presented as a generalization of the power method.

Originally, the idea of the method of subspace iteration has been developed by Bauer, and was known as treppeniteration (staircase iteration). Suppose there is provided a system of $m$ vectors that form the matrix of size $n \times m: X_{0}=\left[x_{1}, \ldots, x_{m}\right]$. The next step is to calculate the matrix

$$
X_{k}=A^{k} X_{0}
$$

for a certain power $k$.

In most cases, if we normalize, as in the power method, the columns vectors all separately from each other, they will all converge to the same eigenvector - the one, which corresponds to the dominant eigenvalue. Thus, the system $X_{k}$ will step by step loose its linear independency. The idea of Bauer's method is to restore the linear independency of these vectors by using the LR or QR factorization.

It is worth mentioning the techniques based on the methods of projection to the Krylov subspaces - another important example of methods for the problems with the matrices of big size. The Krylov subspaces are formed by the iterations of the simple power method. So, if being more precise, these methods provide a possibility to calculate the approximate solution of problem in the following subspace:

$$
K_{m} \equiv \operatorname{span}\left\{v, A v, A^{2} v, \ldots, A^{m-1} v\right\} .
$$

The known examples of this class of methods are the Lanczos algorithms and the Arnoldi methods.

An interesting approach to solve the multi-parameter spectral problem is to calculate the curves of eigenvalues of the matrix of such problem. Starting with a simple pair of eigenvalue and eigenvector, it is computed the curve of eigenvalues to which belongs the starting eigenvalue. After that, by using the 
continuation methods, the other curves of eigenvalues are computed. This method is used, for example, to find the approximate solution of the boundary spectral problem for the differential equation (5).

Continuing the discussion about the eigenvalue problems for the differential equations, it is interesting to mention that there is quite a lot of spectral problems deriving from the discretization of multi-parameter Sturm-Liouville problems for regular differential equations. Naturally, there is a set of methods developed for this type of problems. Some new algorithms are presented in [6]. They are based on the theorem of the matrix trace and on the differential theory of the QR decomposition.

The partial case of multi-parameter eigenvalue problems are the two-dimensional problems:

$$
\begin{aligned}
& A_{1} x=\lambda B_{1} x+\mu C_{1} x, \\
& A_{2} y=\lambda B_{2} y+\mu C_{2} y,
\end{aligned}
$$

where $A_{i}, B_{i}$ and $C_{i}$ are real-values symmetric matrices of size $n_{i} \times n_{i}, i=1,2, \lambda, \mu \in \mathbb{R}^{1}, x \in \mathbb{R}^{n_{1}}$, $y \in \mathbb{R}^{n_{2}}$.

The problems of this type are the matter of analysis widely presented in the literature. It is interesting to mention that most of the existing numerical algorithms for the two-parameter eigenvalue problems $[7,8]$ require the problem to be right-defined.

The problem $(7)$ is called right-defined (see, $[9,10]$ ), if the following condition is fulfilled:

$$
D(x, y) \equiv\left|\begin{array}{ll}
x^{T} B_{1} x & x^{T} C_{1} x \\
y^{T} B_{2} y & y^{T} C_{2} y
\end{array}\right| \geqslant \gamma>0
$$

for all the vectors $x \in \mathbb{R}^{n_{1}}, y \in \mathbb{R}^{n_{2}}$. If the matrices $A_{i}, B_{i}$ and $C_{i}$ are right-defined and symmetric, it means that there exist linearly-independent eigenvectors of size $n_{1}+n_{2}$ of the problem (7).

For the two-parameter spectral problem (7) it is possible to construct an associated eigenvalue problem in the form of coupled system of equations. This require to build the following matrices of the size $N=n_{1} n_{2}$ in the tensor product of the spaces $V=\mathbb{R}^{n_{1}} \otimes R^{n_{2}}$ :

$$
\begin{aligned}
& \Delta_{0}=B_{1} \otimes C_{2}-C_{1} \otimes B_{2}, \\
& \Delta_{1}=A_{1} \otimes C_{2}-C_{1} \otimes A_{2}, \\
& \Delta_{2}=B_{1} \otimes A_{2}-A_{1} \otimes B_{2} .
\end{aligned}
$$

As far as the tensor product of the symmetric matrices is symmetric, the matrices $\Delta_{i}, i=0,1,2$ are symmetric. From the fact that the problem (7) is right-defined, it follows that $\Delta_{0}$ is an inverse matrix, and the matrices $\Delta_{0}^{-1} \Delta_{1}, \Delta_{0}^{-1} \Delta_{2}$ commutate, (see [11]). Such two-parameter problems are called non-singular, and they are equivalent to the associated coupled problem

$$
\Delta_{1} z=\lambda \Delta_{0} z, \quad \Delta_{2} z=\mu \Delta_{0} z
$$

for $z \in V, z=x \otimes y,[12]$.

The coupled problem (8) for the right-defined problem (7) can be solved by using standard numerical methods - for example, the method of simultaneous diagonalization of the associated matrices $[8,13]$. This method is based on the fact that if there exists a complete system of eigenvalues in the space $V$, then there exists a matrix $X$ such that $X^{T} X=I$, and

$$
\begin{aligned}
& X^{T} \Delta_{0}^{-1} \Delta_{1} X=\Lambda=\operatorname{diag}\left\{\lambda_{1}, \lambda_{2}, \ldots, \lambda_{N}\right\}, \\
& X^{T} \Delta_{0}^{-1} \Delta_{2} X=M=\operatorname{diag}\left\{\mu_{1}, \mu_{2}, \ldots, \mu_{N}\right\} .
\end{aligned}
$$

This ensures that the eigenvalues are arranged in a specific order and, thus, allows us to retrieve the required pairs $\left(\lambda_{i}, \mu_{i}\right)$. Some numerical methods are developed for the problems, where the matrices $B_{i}$ and $C_{i}$ are strictly positive or negative: the two-dimensional bisection method by X. Ji [14], or the 
M. Shimasaki's method of continuation by parameter $[15,16]$. The idea of the continuation method is based on the construction of the operator-function

$$
\begin{gathered}
H: \mathbb{R}^{n_{1}} \times \mathbb{R}^{n_{2}} \times \mathbb{R} \times \mathbb{R} \times[0,1] \rightarrow \mathbb{R}^{n_{1}} \times \mathbb{R}^{n_{2}} \times \mathbb{R} \times \mathbb{R}, \\
H(x, y, \lambda, \mu, t) \equiv\left[\begin{array}{c}
(1-t) W_{1} x+t A_{1} x-\lambda B_{1} x-t \mu C_{1} x \\
(1-t) W_{2} y+t A_{2} y-\lambda B_{2} y-t \mu C_{2} y \\
\frac{1}{2}\left(1-x^{T} x\right) \\
\frac{1}{2}\left(1-y^{T} y\right)
\end{array}\right],
\end{gathered}
$$

where the $W_{i}, i=1,2$ are some symmetric matrices of size $n_{1} \times n_{2}$ such that the following eigenvalues problems

$$
\begin{aligned}
& W_{1} x=\lambda B_{1} x, \\
& W_{2} y=\mu C_{2} y
\end{aligned}
$$

have $n_{1}$ and $n_{2}$ different eigenvalues.

Obviously, the solution of the problem $H(x, y, \lambda, \mu, t)=0$ is also the solution of the two-parameter problem

$$
\begin{aligned}
& (1-t) W_{1} x+t A_{1} x=\lambda B_{1} x+t \mu C_{1} x \\
& (1-t) W_{2} y+t A_{2} y=t \lambda B_{2} y+\mu C_{2} y
\end{aligned}
$$

which coincides with the solution of the problem (7) if $t=1$, and with the solution of problem (10)-(11) if $t=0$.

Since the problem (12) is right-defined and has $N$ real-valued eigenvalues for each $t \in[0,1]$, there exists $N$ continuous curves, which can be parameterized by using the parameter $t$ :

$$
v_{i}(t)=\left(x_{i}(t), y_{i}(t), \lambda_{i}(t), \mu_{i}(t), t\right), \quad i=1,2, \ldots, N,
$$

such that $v_{i}(t) \in \Gamma$, where

$$
\Gamma=\left\{(x, y, \lambda, \mu, t) \in \mathbb{R}^{n_{1}} \times \mathbb{R}^{n_{2}} \times \mathbb{R} \times \mathbb{R} \times[0,1] \mid H(x, y, \lambda, \mu, t)=0\right\}
$$

is the set of solutions of the operator-function (9).

To conclude, the continuation method for the construction of the curves $v_{i}(t), i=1,2, \ldots, N$, from $t=0$ till $t=1$ can be implemented by the following algorithm 1 [17].

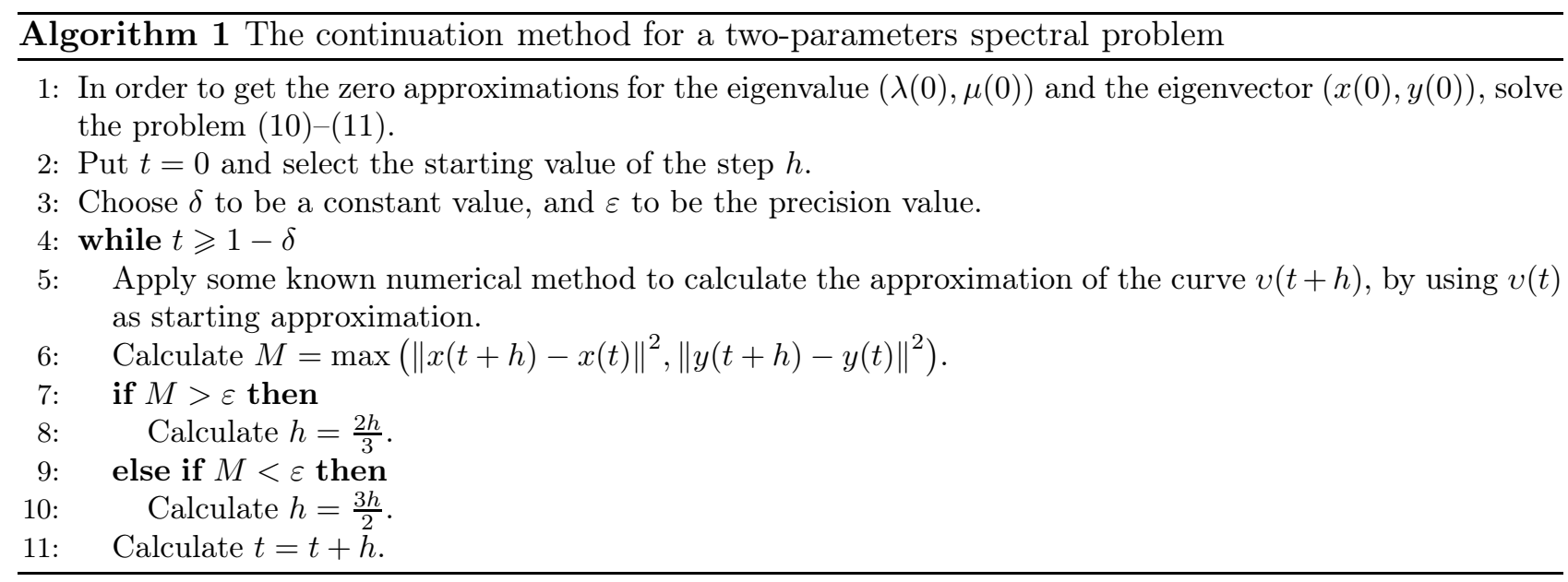


The main principle of the continuation method is based on the property that if the parameter $t$ takes gradual values from the interval $[0,1]$, then $v(t)$ always is a good starting approximation for $v(t+h)$.

In order to be able to gradually build the curve from $t=0$ till $t=1$, in the step 5 of the Algorithm 1 , it is necessary to use a locally convergent numerical method, which would converge to the eigenvalue and the eigenvector of the problem (7). The Newton method can be used as such method. It is applied to the non-linear two-parameter system

$$
F(u) \equiv\left[\begin{array}{c}
A_{1} x-\lambda B_{1} x-\mu C_{1} x \\
A_{2} y-\lambda B_{2} y-\mu C_{2} y \\
\frac{1}{2}\left(x^{T} x-1\right) \\
\frac{1}{2}\left(y^{T} y-1\right)
\end{array}\right]=0, \quad u=(x, y, \lambda, \mu)^{T}
$$

Applying the Newton method to the equation $F(u)=0$, we obtain

$$
F^{\prime}\left(u_{k}\right)\left(u_{k+1}-u_{k}\right)=-F\left(u_{k}\right)
$$

where

$$
F^{\prime}(u)=\left[\begin{array}{cccc}
A_{1}-\lambda B_{1}-\mu C_{1} & 0 & -B_{1} x & -C_{1} x \\
0 & A_{2}-\lambda B_{2}-\mu C_{2} & -B_{2} y & -C_{2} y \\
x^{T} & 0 & 0 & 0 \\
0 & y^{T} & 0 & 0
\end{array}\right] .
$$

We have to solve the following linear system, according to a step of the Newton method (13):

$$
\begin{aligned}
{\left[\begin{array}{cccc}
A_{1}-\lambda_{k} B_{1}-\mu_{k} C_{1} & 0 & -B_{1} x_{k} & -C_{1} x_{k} \\
0 & A_{2}-\lambda_{k} B_{2}-\mu_{k} C_{2} & -B_{2} y_{k} & -C_{2} y_{k} \\
x_{k}^{T} & 0 & 0 & 0 \\
0 & y_{k}^{T} & 0 & 0
\end{array}\right]\left[\begin{array}{c}
\Delta x_{k} \\
\Delta y_{k} \\
\Delta \lambda_{k} \\
\Delta \mu_{k}
\end{array}\right] } \\
\\
=\left[\begin{array}{c}
-\left(A_{1}-\lambda_{k} B_{1}-\mu_{k} C_{1}\right) x_{k} \\
-\left(A_{2}-\lambda_{k} B_{2}-\mu_{k} C_{2}\right) y_{k} \\
\frac{1}{2}\left(1-x_{k}^{T} x_{k}\right) \\
\frac{1}{2}\left(1-y_{k}^{T} y_{k}\right)
\end{array}\right]
\end{aligned}
$$

and, then, after calculating the new approximations,

$$
\begin{aligned}
x_{k+1} & =x_{k}+\Delta x_{k}, \\
y_{k+1} & =y_{k}+\Delta y_{k}, \\
\lambda_{k+1} & =\lambda_{k}+\Delta \lambda_{k}, \\
\mu_{k+1} & =\mu_{k}+\Delta \mu_{k},
\end{aligned}
$$

from the (14) we obtain

$$
\begin{gathered}
\left(A_{1}-\lambda_{k} B_{1}-\mu_{k} C_{1}\right) x_{k+1}=\Delta \lambda_{k} B_{1} x_{k}+\Delta \mu_{k} C_{1} x_{k}, \\
\left(A_{2}-\lambda_{k} B_{2}-\mu_{k} C_{2}\right) y_{k+1}=\Delta \lambda_{k} B_{2} y_{k}+\Delta \mu_{k} C_{2} y_{k}, \\
x_{k}^{T} x_{k+1}=\frac{1}{2}\left(x_{k}^{T} x_{k}+1\right) \\
y_{k}^{T} y_{k+1}=\frac{1}{2}\left(y_{k}^{T} y_{k}+1\right) .
\end{gathered}
$$


For simplicity let us introduce the denotations:

$$
\begin{aligned}
\nu_{k} & =\left(A_{1}-\lambda_{k} B_{1}-\mu_{k} C_{1}\right)^{-1} B_{1} x_{k}, \\
w_{k} & =\left(A_{1}-\lambda_{k} B_{1}-\mu_{k} C_{1}\right)^{-1} C_{1} x_{k}, \\
p_{k} & =\left(A_{2}-\lambda_{k} B_{2}-\mu_{k} C_{1}\right)^{-1} B_{2} y_{k}, \\
q_{k} & =\left(A_{2}-\lambda_{k} B_{2}-\mu_{k} C_{2}\right)^{-1} C_{2} y_{k},
\end{aligned}
$$

and multiply the expressions (17) by $x_{k}^{T}$ and $y_{k}^{T}$.

This way, we obtain the linear system

$$
\left[\begin{array}{cc}
x_{k}^{T} \nu_{k} & x_{k}^{T} w_{k} \\
y_{k}^{T} p_{k} & y_{k}^{T} q_{k}
\end{array}\right]\left[\begin{array}{c}
\Delta \lambda_{k} \\
\Delta \mu_{k}
\end{array}\right]=\left[\begin{array}{c}
\frac{1}{2}\left(x_{k}^{T} x_{k}+1\right) \\
\frac{1}{2}\left(y_{k}^{T} y_{k}+1\right)
\end{array}\right]
$$

for $\Delta \lambda_{k}$ and $\Delta \mu_{k}$. By solving this system, we calculate the values $x_{k+1}$ and $y_{k+1}$ from (15)-(16).

To finalize, let us present the algorithm of the Newton method for the right-defined two-parameters spectral problem.

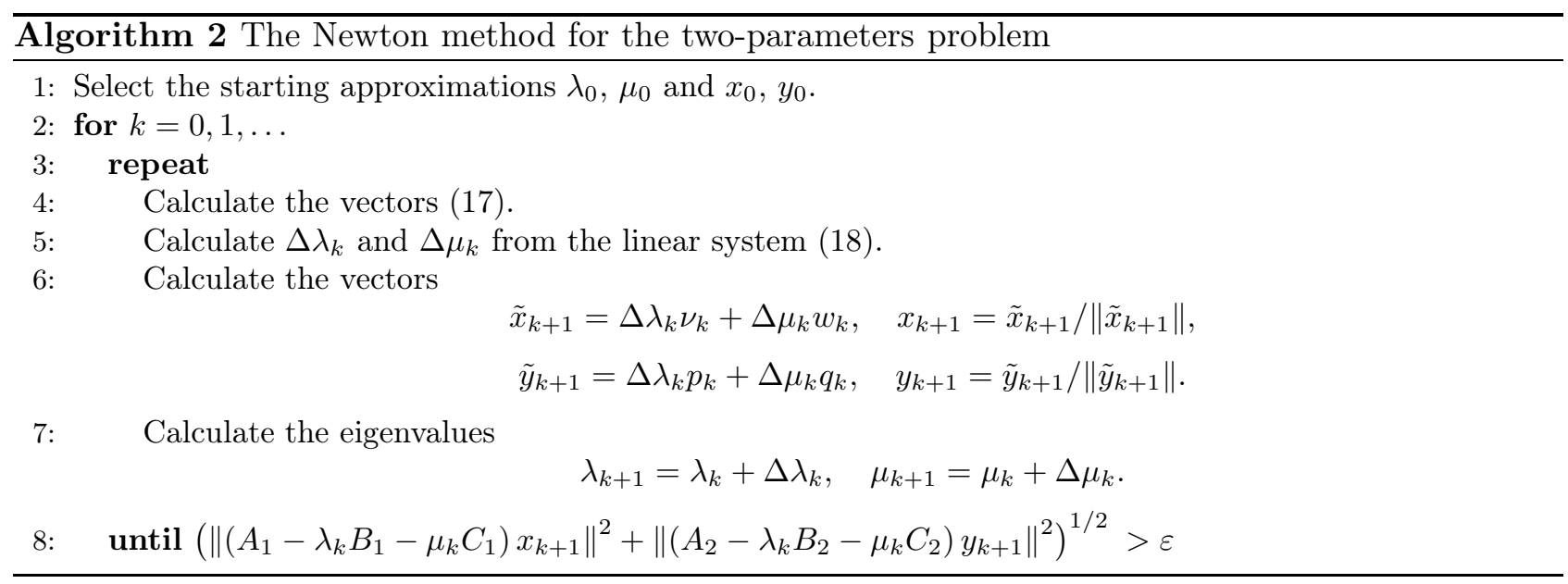

We made a short overview of the practical applications of the multi-parameter spectral problem and of some most known numerical methods and approaches to this problem. This list is not complete as the analysis works are still ongoing and the new, more efficient methods, are being developed.

[1] Saad Y. Numerical methods for large eigenvalue problems. Second edition. SIAM (2011).

[2] Hochstenbach M.E., Plestenjak B. Harmonic Rayleigh-Ritz for the Multiparameter eigenvalue problem. Department of Mathematics and Computing Science, Eindhoven University of Technology (2006).

[3] Atkinson F. V. Multiparameter spectral theory. Bull. Amer. Math. Soc. 74 (1), 1-27 (1968).

[4] Müller R. E. Numerical solution of multiparameter eigenvalue problems. ZAMM. 62 (12), 681-686 (1982).

[5] Molzahn D. Power system models formulated as eigenvalue problems and properties of their solutions A thesis for the degree of master of science. University of Wisconsin-Madison (2010).

[6] DaiH. Numerical methods for solving multiparameter eigenvalue problems. International Journal of Computer Mathematics. 72, 331-347 (1999).

[7] Blum E. K., Curtis A. R. A convergent gradient method for matrix eigenvector-eigentuple problems. Numer. Math. 31 (3), 247-263 (1978).

[8] Slivnik T., Tomšič G. A numerical method for the solution of two-parameter eigenvalue problem. J. Comp. Appl. Math. 15 (1), 109-115 (1986).

[9] Sleeman B. D. Multiparameter spectral theory in Hilbert space. London, San Francisco, Melbourne, Pitnam Press (1978). 
[10] Volkmer H. Multiparameter eigenvalue problems and expansion theorem. Lect. Notes Math. 1336 (1988).

[11] Atkinson F. V. Multiparameter Eigenvalue Problems. Matrices and Compact Operators. Vol.1. New York, London, Academic Press (1972).

[12] Fox L., Hayes L., Mayers D. F. The double eigenvalue problem. John J. H. Miller (Ed.), Topics in Numerical Analysis, Proceedings of the Royal Irish Academy Conference on Numerical Analysis, 1972, Academic Press, New York (1973), pp. 93-112.

[13] JiX. Numerical solution of joint eigenpairs of a family of commutative matrices. Appl. Math. Lett. 4 (3), 57-60 (1991).

[14] Ji X. A two-dimensional bisection method for solving two-parameter eigenvalue problems. SIAM J. Matrix Anal. Appl. 13 (4), 1085-1093 (1992).

[15] Shimasaki M. Homotopy algorithm for two-parameter eigenvalue problems. Trans Japan SIAM. 5, 121129 (1995).

[16] Shimasaki M. Numerical method based on homotopy algorithm for two-parameter problems. Trans Japan SIAM. 6, (1996).

[17] Plestenjak B. A. A continuation method for a right definite two-parameter eigenvalue problem. SIAM J. Matrix. Anal. Appl. 21 (4), 1163-1184 (2000).

\title{
Огляд чисельних методів відшукання наближеного розв'язку багатопараметричних задач на власні значення
}

\author{
Ярошко С. ${ }^{1}$, Ярошко O. ${ }^{2}$ \\ ${ }^{1}$ Начіональний університет «Львівська політехніка» \\ вул. С. Бандери, 12, 79013, Львів, Україна \\ 2 Львівсъкий начіональний університет імені Івана Франка \\ вул. Університетсъка, 1, 79000, Лъвів, Україна
}

Метою цієї роботи є огляд наявних чисельних методів та технік розв'язування багатопараметричної задачі на власні значення. Подано також відомі приклади застосування багатопараметричної спектральної задачі.

Ключові слова: власне значення, власний вектор, задача на власні значення, багатопараметрична спектральна задача, чисельні методи.

2000 MSC: 34B09, 34L15, 34L16, 58C40, 65F 15

УдК: 519.6 